\title{
Isolation and phenotypic characterization of side population cells in oral squamous cell carcinoma
}

\author{
YUTAO LIU ${ }^{1}$, PINGPING CUI ${ }^{2}$, JIMING CHEN ${ }^{1}$ and WANSHAN LI ${ }^{1}$ \\ ${ }^{1}$ Department of Oral and Maxillofacial Surgery, Children's Hospital, Chongqing Medical University, Chongqing 400014; \\ ${ }^{2}$ Department of Stomatology, Central Hospital, Shengli Oil Field, Dongying, Shandong 257000, P.R. China
}

Received January 17, 2014; Accepted August 11, 2014

DOI: $10.3892 / \mathrm{mmr} .2014 .3133$

\begin{abstract}
A subset of cancer stem cells termed 'side population' (SP) cells has been identified in several types of solid tumor and is known to be responsible for the failure of chemotherapy and for cancer relapse. In the present study, the oral squamous cell carcinoma (OSCC) cell line SCC-55 was used for analysis of the SP cells based on Hoechst 33342 exclusion, via the ATP-binding cassette (ABC) transporter, with and without verapamil using fluorescence activated cell sorting. The results demonstrated that the presence of SP cells in the SCC-55 cell line was $2.8 \%$, which was reduced to $0.6 \%$ in the presence of verapamil, an inhibitor of the ABC transporter. In addition, following exposure to the chemotherapeutic drug 5-fluorouracil, a high survival rate was observed in the SP cells due to overexpression of the ABCG2 and B-cell lymphoma 2 proteins. In addition, overexpression of stem cell surface markers, including CD44 and CD147 was observed in the SP cells. Therefore, these findings suggested that OSCC contains SP cells, which share certain characteristics with stem cells. The coexpression of ABC transporters and stem cell surface markers in SP cells may be associated with resistance to chemotherapeutic agents and also supports a role for these cells in tumor recurrence, metastasis and invasion.
\end{abstract}

\section{Introduction}

Globally, oral cancer is the sixth most common type of cancer affecting $3 \%$ of the world's population. There has been an increase in the rate of occurrence and, in 2007, this rate increased over $11 \%$ in one year (1). Annually, almost $7 \%$ of cancer-associated mortality in males and $4 \%$ in females is attributed to oral cancer (1). Tobacco use, smoking, alcohol and areca nut chewing

Correspondence to: Dr Wanshan Li, Department of Oral and Maxillofacial Surgery, Children's Hospital, Chongqing Medical University, 136 Zhongshan Road, Yuzhong, Chongqing 400014, P.R. China

E-mail:wanshanli2@gmail.com

Key words: cancer stem cells, side population, transporters, multidrug resistance, chemotherapy are proposed as possible risk factors for oral cancer (1). Oral cancer can affect any part of the oral cavity, including the lips, tongue, mouth and throat, which can be categorized into two types. Oral cavity cancer develops in the mouth itself, whereas oropharyngeal cancer develops in the oropharynx, which is located in the throat just behind the mouth. The most common type of oral cancer is squamous cell carcinoma (SCC), which affects the tissue lining the oral cavity $(2,3)$.

Despite advances in the treatment of cancer, the clinical outcome is far from expected and recurs in patients even following treatment. It has been found that treatment failure, or minimal residual disease, occurs due to the persistence of cancer stem cells (CSCs) that evade the treatment regimen (4,5). The population of CSCs possesses characteristics associated with stem cells, including self-renewal and exhibits high in vivo tumorigenicity, differentiation potential as well as multidrug and apoptotic resistance (6). The difficulty in eradicating tumor tissue may be due to conventional treatments targeting the bulk of the tumor cells, but not the CSCs, which maintain the tumor tissue.

Cells that exclude Hoechst 33342 dye are referred to as side population (SP) cells. These cells share several characteristics with CSCs; in particular, they possess the capacity for tumor initiation, express stem-like genes and demonstrate resistance to chemotherapeutic drugs. Therefore, the dye exclusion technique is valuable in assisting in the identification of a unique population of cells with stem-like characteristics (7). SP cells also overexpress ATPase binding cassette $(\mathrm{ABC})$ transporters, including $\mathrm{ABCB} 1$, also termed multidrug resistance protein 1 (MDR1), ABCC1 and ABCG2, also termed breast cancer resistance protein 1 , which contribute to multidrug resistance and also express stem cell surface markers, including CD133, CD44, CD34, CD29 and CD24. SP cells have also been identified in several types of solid tumor, including breast cancer (4), brain tumor (5), glioblastoma (5), gastrointestinal tumor (6), head and neck squamous cell carcinoma (7) as well as in hepatocellular cell lines (4) and in primary cultures from patients with neuroblastoma (8).

Therefore, the sorting of SP cells assists in identifying cancerous stem cells and its subsequent characterization substantiates the mechanism underlying oncogenesis and drug resistance (9). Consequently, the present study investigated the prevalence of SP cells using an established oral 
squamous cell carcinoma (OSCC) cell line SCC-55, based on Hoechst 33342 efflux. In addition, the isolated SP cells were characterized for the presence of cell surface markers, including CD147, CD44 and CD29.

\section{Materials and methods}

Cell line and cell culture. The SCC-55 human cell line was obtained from cancerous growth in the mandibular region (grade three; recurrent type; American Type Cell Culture, Manasas, VA, USA). The SCC-55 cell lines were cultured in Dulbecco's modified Eagle's medium (DMEM; Gibco-BRL, Carlsbad, CA, USA) with $10 \%$ fetal bovine serum (Gibco-BRL) supplemented with antibiotics and maintained in T-75 flasks at $37^{\circ} \mathrm{C}$ in a humidified $5 \% \mathrm{CO}_{2}, 95 \%$ air atmosphere. When the SCC-55 cells attained $90 \%$ confluence, they were removed from the culture flask using Trypsin-EDTA (0.25\% 53 mM EDTA; Sigma, St. Louis, MO, USA), washed in phosphate-buffered saline (PBS), suspended in 10\% DMEM and centrifuged at 4,000 $\mathrm{x}$ g for $6 \mathrm{~min}$. The cells were then resuspended in 10\% DMEM and cells were counted using a hemocytometer (Thermo Fisher Scientific, Waltham, MA, USA). The present study was approved by the ethics committee of Chongqing Medical University (Chongqing, China).

Study group. The control group consisted of cells + Hoechst $33342(\mathrm{n}=5)$. The drug treated group consisted of cells + verapamil + Hoechst $33342(\mathrm{n}=5)$.

Labeling with Hoechst 33342 . The cells in the staining medium $\left(\sim 10^{6}\right.$ cells $/ \mathrm{ml}$ of $10 \%$ DMEM $)$ were labeled with Hoechst 33342 stock (Sigma) bis-benzimide $(5 \mu \mathrm{l} / \mathrm{ml})$ with either the dye alone or in combination with verapamil $(0.8 \mu /$ $\mathrm{ml})$. The cells were mixed and placed in a water bath at $37^{\circ} \mathrm{C}$ for $90 \mathrm{~min}$. After $90 \mathrm{~min}$, the cells were centrifuged $\left(1,500 \mathrm{xg}\right.$ for $10 \mathrm{~min}$ at $\left.4^{\circ} \mathrm{C}\right)$ and resuspended in $500 \mu 1$ Hank's balanced salt solution containing $10 \mathrm{mM}$ 2-[4-(2-hydroxyethyl)piperazin-1-yl]ethanesulfonic acid. Finally, the cells were counterstained using propidium iodide (PI; $2 \mu \mathrm{g} / \mathrm{ml}$ ) at $4^{\circ} \mathrm{C}$. The cells were filtered through a $50 \mu \mathrm{m}$ nylon mesh (BD Biosciences, Franklin Lakes, NJ, USA) to remove clumps of cells into labeled fluorescence-activated cell sorting (FACS) tubes. Separate tubes containing 10\% DMEM were used for sterile sorting of the SP cells and the main population cells. The cells were sorted using a flow cytometer (FACS Aria II; BD Biosciences). The Hoechst 33342 dye was excited at $355 \mathrm{~nm}$ and its dual-wavelength fluorescence was analyzed using a flow cytometer (blue, $450 \mathrm{~nm}$; red, $675 \mathrm{~nm}$; FACS Aria II; BD Biosciences, Franklin Lakes, NJ, USA).

In vitro proliferation assay. The sorted SP and non-SP cells were inoculated into a 96-well plate at $2 \times 10^{6}$ cells/well and then cultured in a $\mathrm{CO}_{2}$ incubator. Each group was set up in triplicate. Cell proliferation was measured every day for 5 days. Each well was supplemented with Cell Counting kit-8 (CCK-8; Tiangen, Beijing, China) solution (10 $\mu \mathrm{l})$ and incubated in a $\mathrm{CO}_{2}$ incubator for $3 \mathrm{~h}$. The optical density (OD) was determined at $450 \mathrm{~nm}$. These data were used to calculate the cell growth based on the mean value of $\mathrm{OD}_{450}$ and the standard deviation values for each well.
Cell resistance experiment. The obtained SP and non-SP cells were cultured in 96-well plates at a concentration of $1 \times 10^{3}$ cells/plate. After $24 \mathrm{~h}, 5$-fluorouracil $(5-\mathrm{FU})$ was added to all cultures to produce a final concentration of $10 \mu \mathrm{g} / \mathrm{ml}$. The plates were then placed in a hatch box for $48 \mathrm{~h}$. Each well was then supplemented with CCK-8 (10 $\mu \mathrm{l})$ solution and the plates were incubated for $3 \mathrm{~h}$. The mean value of $\mathrm{OD}_{450}$ was obtained and is presented as a graph. Cell resistance was calculated using the following formula: Cell resistance rate $(\%)=\left(\right.$ experimental group $\mathrm{OD}_{450} /$ control group $\left.\mathrm{OD}_{450}\right)$ x 100.

Immunocytochemistry. The sorted SP cells and main population cells were seeded in $35 \mathrm{~mm}$ culture plates $(\sim 100 \mu \mathrm{l})$ and incubated for $3 \mathrm{~h}$, following which $1 \mathrm{ml}$ of medium (10\% DMEM) was added. Following incubation overnight, the cells were rinsed with PBS and fixed in 4\% paraformaldehyde in 1X PBS for $5 \mathrm{~min}$ at $4^{\circ} \mathrm{C}$. The cells were then washed with $1 \mathrm{X}$ PBS and blocked with $1 \%$ bovine serum albumin (BSA)-Tris-buffered saline (TBS) with RNase (10 $\mu \mathrm{l} / 1,000 \mu \mathrm{l}$ of 3\% BSA-TBS). After $1 \mathrm{~h}$ incubation at room temperature, the cells were washed with PBS and then incubated with the primary antibodies, mouse anti-human CD147 monolclonal antibody (Santa Cruz Biotechnology, Inc., Dallas, TX, USA) and mouse anti-human CD44 monoclonal antibody (Santa Cruz Biotechnology, Inc.), in $1 \%$ BSA-TBS $(1: 100 ; 2 \mu \mathrm{l} / 200 \mu \mathrm{l})$ prior to incubation overnight at $4^{\circ} \mathrm{C}$. Following washing with $1 \mathrm{X}$ PBS, the cells were incubated with a rabbit anti-mouse polyclonal secondary antibody conjugated with fluorescein isothiocyanate (1:100 in 1\% BSA-TBS; Santa Cruz Biotechnology, Inc.) at room temperature for $1 \mathrm{~h}$. The cells were then washed with PBS and PI was added $(1 \mu \mathrm{l} / 200 \mu \mathrm{l}$ PBS). The cells were viewed under a confocal laser scanning microscope (Leica TCS; Leica, Mannheim, Germany). Image analysis and preparation of figures was performed using Adobe Photoshop CS4 software (Adobe Systems, Inc., San Jose, CA, USA).

Biochemistry. For western blot analysis, proteins were extracted from the SP and non-SP cells and the protein concentration was determined using a Bradford assay. Following sodium dodecyl sulfate-polyacrylamide gel electrophoresis and transfer onto a membrane, the gels were treated with the primary antibodies rabbit anti-human ABCG2, CD147, CD44 and B-cell lymphoma 2 (Bcl-2), the secondary antibody (goat anti-rabbit IgG with alkaline phosphatase markers) and a chemiluminescence reagent. Blots were detected and scanned using a densitometer (Bio-Rad GS-710; Bio-Rad, Hercules, CA, USA).

\section{Results}

FACS analysis of SP cells containing MDRI using Hoechst 33342. In FACS analysis, cells or particles passing through the laser beam scatter light. This is detected as forward scatter, which correlates with cell size, and side scatter, which is dependent on cell or particle density. The density may include the number of granules in the cytoplasm or the size of the membrane. Populations of cells are often determined based on their different size and density. Live cells were selected against PI as a P1 gated population. PI was used to exclude dead cells 


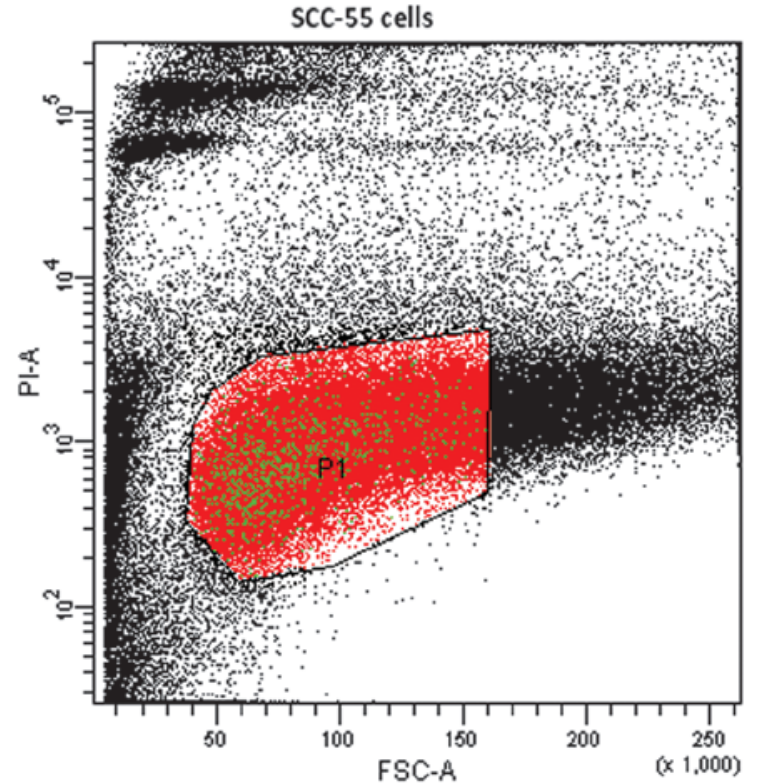

Figure 1. A representative dot plot produced from fluorescence-activated cell sorting analysis showing the presence of live cells in the $\mathrm{P} 1$ gated population. Cells were stained using propidium iodide to exclude the dead cells. SCC, squamous cell carcinoma; FSC, forward scatter.

from the sample (Fig. 1). The SP cells were sorted from the gated P1 cells using Hoechst 33342, a DNA binding dye. By simultaneously monitoring the fluorescence emission of Hoechst 33342 at $450 \mathrm{~nm}$ (SP-Violet) and at $675 \mathrm{~nm}$ (SP-Red) following ultraviolet excitation, a set of cells were observed, which exhibited low blue and red fluorescence. The gate was set according to the ability of cells to efflux Hoechst 33342 and to the sensitivity of the cells to verapamil. In the FACS analysis, the lower left quadrant was designated SP and was inhibited by verapamil. The upper right quadrant was designated as MP, in which Hoechst red was observed and was not inhibited by verapamil (Fig. 2A and B).

SP cells are a distinct population of $\mathrm{P} 2$ gated cells located towards the SP-violet region of the FACS profile dot plot. The active exclusion of Hoechst 33342 by SP cells (Fig. 2A) involves the $\mathrm{ABC}$ transporter transmembrane protein MDR1. The number of cells collected $(\sim 59,000)$ comprised $\sim 55 \%$ of the initial cell count. Of the $59 \times 10^{3}$ cells (P1) analyzed, Hoechst dye was effluxed by $2.8 \%$ cells in the $\mathrm{P} 2$ gated region (Fig. 2A). The verapamil-treated cells of the same cell line were sorted as described previously with Hoechst 33342. A total of $\sim 53,199$ P1 cells were obtained, which was $\sim 44 \%$ of the initial cell count. Following treatment with verapamil, SP cells were reduced from 2.8 to $0.6 \%$. Verapamil is an MDR1 transporter protein inhibitor, which inhibits the drug efflux action of cells (Fig. 2B). Therefore, treatment with verapamil significantly reduced the proportion of SP cells.

Characterization of SP cells. The SP and non-SP cells sorted by FACS were subject to further phenotypic characterization, including cell proliferation and cell survival assays. The growth rates of SP and non-SP cells are shown in Fig. 3. Rapid proliferation was observed in the SP cells starting from day (D)3 and became more confluent on D7 (data not shown). However, the growth rate of the non-SP cells was
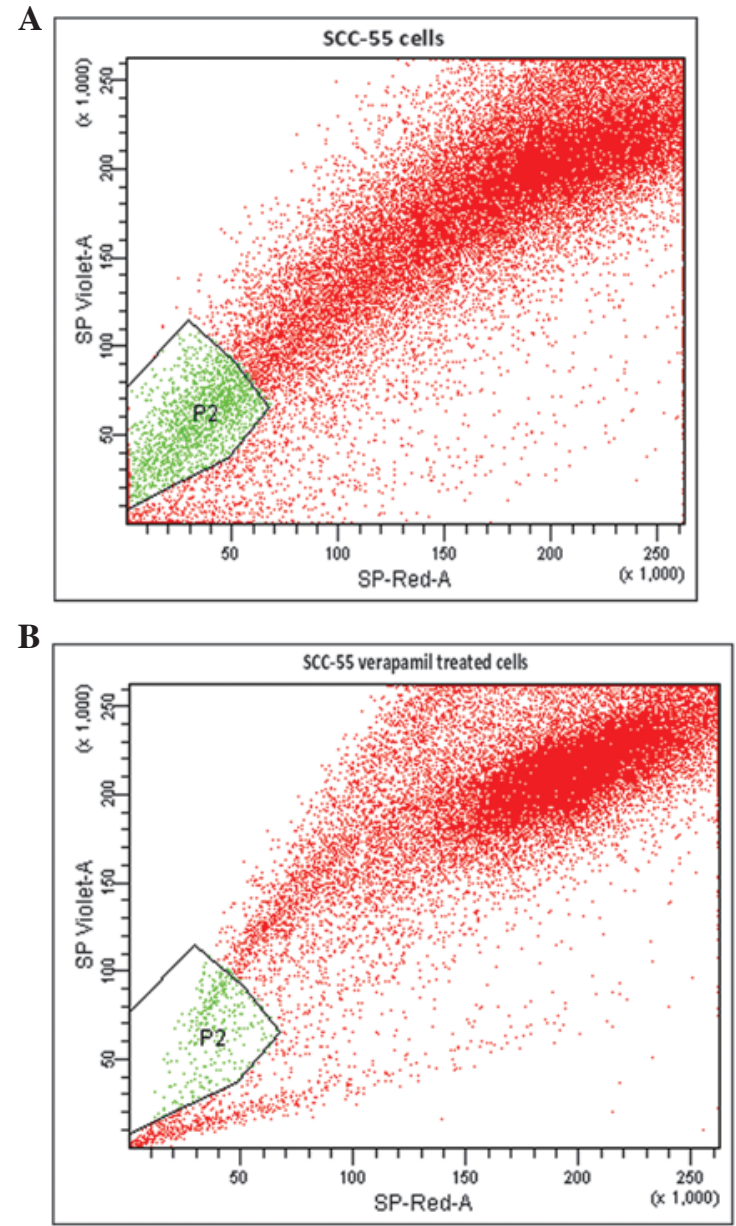

Figure 2. Analysis of SP cells in the oral squamous cancer cell line SCC-55. (A) SCC-55 cells were stained using a Hoechst 33342 dye and analyzed using flow cytometry. The SP cells are outlined (P2 gated population) and are presented as a percentage of the total cell population. (B) In the presence of verapamil, the percentage of SP cells was significantly reduced. SP, side population; SCC, squamous cell carcinoma.

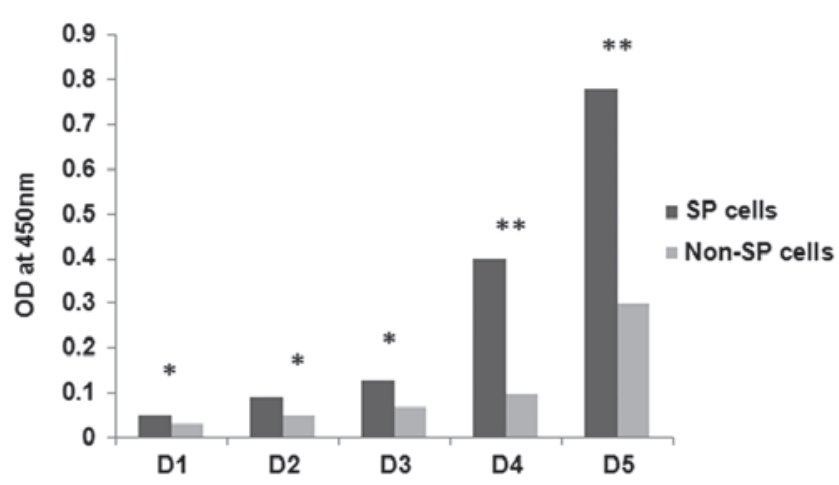

Figure 3. Cell proliferation rate for SP and non-SP cells. The $\mathrm{x}$-axis indicates time, the $y$-axis indicates the corresponding OD value at $450 \mathrm{~nm}$. $\mathrm{P}<0.05$ and ${ }^{* *} \mathrm{P}<0.01$ SP vs. non-SP cells. D, day; SP, side population; OD, optical density.

significantly lower when compared with the SP cells (Fig. 3). The growth rate of the SP cells was significantly higher compared with that of the non-SP cells $\left({ }^{*} \mathrm{P}<0.05 ;{ }^{* *} \mathrm{P}<0.01\right)$. Following the in vitro proliferation assays, the survival rates of the SP cells were analyzed following exposure to $10 \mu \mathrm{g} / \mathrm{ml}$ 5-FU. As shown in Fig. 4, SP cells demonstrated a signifi- 


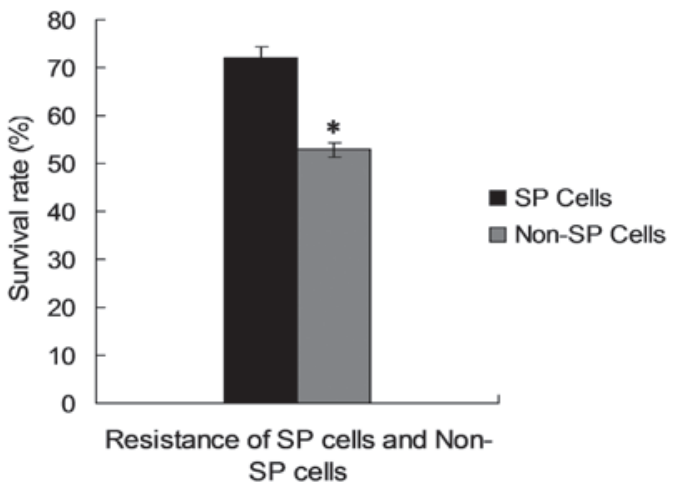

Figure 4. Resistance of SP cells and non-SP cells to 5-FU. The y-axis indicates the survival rate of the two groups following treatment with $10 \mu \mathrm{g} /$ $\mathrm{ml}$ 5-FU for $3 \mathrm{~h} .{ }^{*} \mathrm{P}<0.05$ SP vs. non-SP cells. 5-FU, 5-fluorouracil; SP, side population.
A
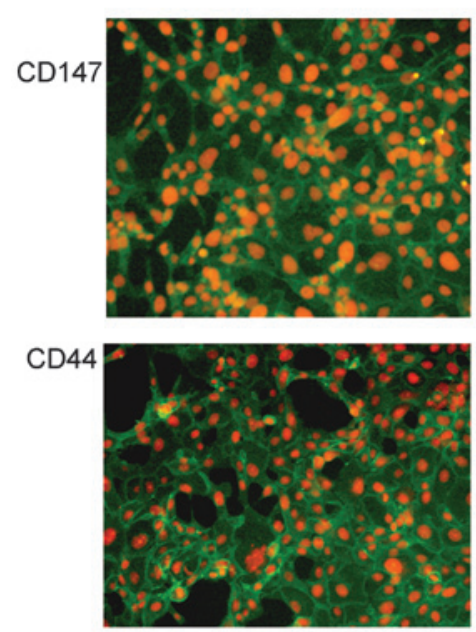

Non-SP
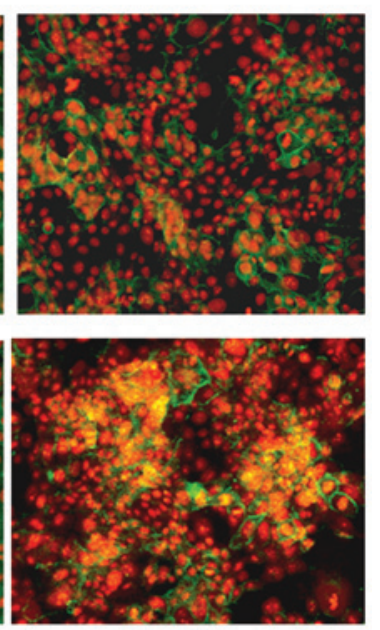

B

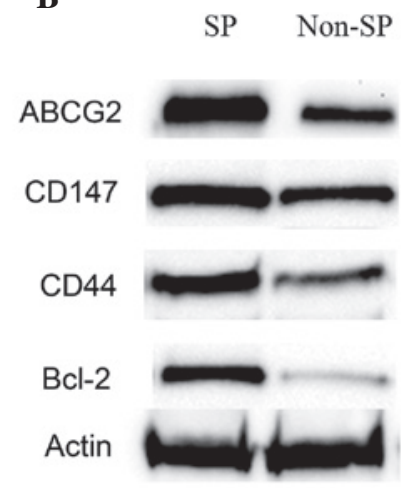

Figure 5. (A) Analysis of cell surface markers by fluorescence microscopy. SP cells (almost entire population) demonstrated positive fluorescence for the presence of CD147 and CD44, however, this fluorescence was not enriched in the Non-SP cells. (B) Western blot analysis of protein expression levels in the SP and non-SP cells. Equal concentrations of protein were loaded in each lane. SP, side population; Bcl-2, B-cell lymphoma 2; ABC, ATP-binding cassette.

cantly higher resistance to 5-FU (73\%) compared with the non-SP cells $\left(52 \%\right.$; $\left.{ }^{*} \mathrm{P}<0.05\right)$.

Furthermore, the isolated SP cells were analyzed for the cell surface markers CD147 and CD44 using antibodies against them. Notably, analysis using a fluorescence microscope (TCS SP2; Leica, Mannheim, Germany) revealed that the SP cells were more positive for the cell surface proteins, including CD147 and CD44 (Fig. 5A) compared with the non-SP cells. This was further confirmed by western blot analysis. CD147 and CD44 were highly expressed in the SP cells, whereas the levels of these proteins were reduced in the non-SP cells (Fig. 5B). The results also demonstrated that the levels of ABCG2 and Bcl-2 expression were higher in the SP cells compared with the non-SP cells (Fig. 5B).

Therefore, the results of the present study suggested that the expression of CD147/CD44, ABCG2 and Bcl-2 is elevated in SP cells and they may act co-operatively as an important factor in drug resistance and in the marked proliferation of cancer cells and tumor invasion.

\section{Discussion}

Traditional treatments have been developed and assayed based mainly on their ability to destroy the majority of the tumor population. However, these treatments may miss CSCs, which are more resistant to chemotherapeutic drugs. These CSCs are responsible for tumor growth, metastasis and relapse (10). There is evidence in several different types of cancer indicating that CSCs survive conventional anticancer therapies that target only the rapidly dividing cells (11) and is associated with treatment failure and tumor recurrence. Therefore, the elimination of CSCs is an important goal for eradicating types of refractory cancer and in providing long-term disease-free survival. To enable this, certain stem cell characteristics can be used to identify CSCs, one of which is the capacity of cells to extrude dyes, including Hoechst 33342. Cells that exclude this dye are termed SP cells (12). SP cells share characteristics of CSCs, in particular they have increased tumor-initiating capacity, express stem-like genes and are resistant to chemotherapeutic drugs. Therefore, the exclusion of dyes is a valuable technique as it enables the identification of this unique population of cells with stem-like characteristics.

In the present study, CSC cells from the OSCC SCC-55 cell line were identified and isolated. SP cells were sorted from the gated P1 cells based on the efflux of Hoechst 33342, a DNA binding dye, using FACS analysis. The exclusion of Hoechst 33342 by SP cells is an active process involving MDR1, a member of the ABC transporter transmembrane proteins. Following treatment with verapamil, the SP cells were reduced from 2.8 to $0.6 \%$ (Fig. $2 \mathrm{~A}$ and B). Verapamil is an MDR1 transporter protein inhibitor, which inhibits the drug efflux action of the cells. Its use also provided confirmation of the presence of the MDR1 protein in the SCC-55 cell line and explained the chemoresistance and tumor recurrence in the mandibular origin from which the cell line was established. The multidrug resistance demonstrated by these cells enabled them to survive the chemotherapy regimen and resulted in a poor prognosis of the patients suffering from oral cancer. The sorted SP cells were further characterized by cell proliferation and cell survival assays. These cells demonstrated increased proliferation capacity and resistance to 5-FU (Figs. 3 and 4). The resistance to 5 -FU was most likely facilitated by two major mechanisms, the activation of $\mathrm{ABC}$ transporters, which expel 
harmful materials from cells and the suppression of apoptosis. Similar to previous findings in gastric cancer cell lines (13), the results of the present study suggested that these processes may have occurred in the SP cells, where the expression of the $\mathrm{ABC}$ transporter protein, $\mathrm{ABCG}$, and the anti-apoptotic protein $\mathrm{Bcl}-2$ were significantly higher compared with the non-SP cells. Collectively, these findings suggested that the chemotherapy resistance characteristics of SP cells were derived mainly from the overexpression of the chemotherapy resistant channel protein ABCG2.

In addition, immunocytochemical analysis was performed on the sorted SCC-55 SP cells to investigate the presence of cell surface markers. Using fluorescence microscopy, almost the entire population of SP cells was more positive to CD147 and CD44 compared with those in the non-SP population (Fig. 5A). Previous studies in different types of solid tumor also revealed that an increased quantity of CD147 on the surface of tumor cells induces tumor invasion and stimulates the production of a number of matrix metalloproteinases by adjacent stromal cells (14). Similarly, in head and neck squamous cell carcinoma, $\mathrm{CD} 44^{+}$cells were enriched with tumorigenic CSCs able to propagate tumor formation in mice, whereas CD44- cells were not (15). Taken together, the findings of the present study suggested that the coexpression of CD147 and CD44 by SP cells positive to MDR1 in SCC-55 indicate a role for these cells in tumor growth, metastasis and recurrence in the mandibular origin from which the recurrent tumor SCC-55 cell line was established. There may be close interaction between CD147, CD44 and MDR1 resulting in the marked augmentation of tumor metastasis, invasion and chemoresistance. Therefore, the characterization of SP cells assists in designing novel therapeutic agents, which selectively target CSCs and may improve cancer therapy by inhibiting ABC transporters. Furthermore, differences between the signaling pathways in normal cells and CSCs require elucidation to provide new therapeutic targets, with the eventual goal of eliminating residual disease and disease recurrence.

\section{References}

1. Ma JL, Zhang L, Brown LM, et al : Fifteen-year effects of helicobacter pylori, garlic, and vitamin treatments on gastric cancer incidence and mortality. J Natl Cancer Inst 104: 488-492, 2012.

2. Ackerman LV: Verrucous carcinoma of the oral cavity. Surgery 23: 670-678, 1948.

3. Crissman JD and Zarbo RJ: Dysplasia in situ carcinoma and progression to invasive squamous cell carcinoma of the upper aerodigestive tract. Am J Surg Pathol 13: 5-16, 1989.

4. Challen GA and Little MH: A side order of stem cells: the SP phenotype. Stem Cells 24: 3-12, 2006.

5. Ramachandran $\mathrm{C}$ and Melnick SJ: Multidrug resistance in human tumors molecular diagnosis and clinical significance. Mol Diagn 4: 81-94, 1999.

6. Ford JM and Hait WN: Pharmacology of drugs that alter multidrug resistance in cancer. Pharmacol Rev 42: 155-199, 1990.

7. Hirschmann-Jax C, Foster AE, Wulf GG, et al: A distinct 'side population' of cells with high drug efflux capacity in human tumor cells. Proc Natl Acad USA 101: 14228-14233, 2004.

8. Robey RW, Shukla S, Finley EM, Oldham RK, et al: Inhibition of Pgp (ABCB1) and MDR associated protein-1 (ABCC1) mediate transport by the orally administered inhibitor CBT-1. Biochem Pharmacol 75: 1032-1042, 2008.

9. Kondo T, Setoguchi T and Taga T: Persistence of a small subpopulation of cancer stem-like cells in the C6 glioma cell line. Proc Natl Acad Sci USA 101: 781-786, 2004.

10. Gil J, Stembalska A, Pesz KA and Sasiadek MM: Cancer stem cells: the theory and perspectives in cancer therapy. App Genet 49: 193-199, 2008.

11. Komuro H, Saihara R, Shinya M, Takita J, et al: Identification of side population cells (stem-like cell population) in pediatric solid tumor cell lines. J Pediatr Surg 42: 2040-2045, 2007.

12. Cho RW and Clarke FM: Recent advances in cancer stem cells. Curr Opin Genet Dev 18: 48-53, 2008.

13. Li R, Wu X, Wei H and Tian S: Characterization of side population cells isolated from the gastric cancer cell line SGC-790. Oncol Lett 5: 877-883, 2013.

14. Guo H, Li R, Zucker S and Toole BP: EMMPRIN (CD147), an inducer of matrix metalloproteinase synthesis, also binds interstitial collagenase to the tumor cell surface. Cancer Res 60: 888-891, 2000.

15. Yanamoto S, Kawasaki G, Yoshitomi I, Iwamoto T, et al: Clinicopathologic significance of EpCAM expression in squamous cell carcinoma of the tongue and its possibility as a potential target for tongue cancer gene therapy. Oral Oncol 43: 869-877, 2007. 